\title{
The Fundamental Solution of Mindlin Plates with Damping in the Laplace Domain and its Applications
}

\author{
P.H. Wen ${ }^{a^{*}}$, M. Adetoro ${ }^{a}, Y . X u^{b}$ \\ ${ }^{a}$ School of Engineering and Materials Science, Queen Mary, University of London, \\ London E1 4NS, UK \\ ${ }^{b}$ School of Aerospace, Automotive and Design Engineering, University of \\ Hertfordshire, AL10 9AB, UK
}

\begin{abstract}
In this paper, a fundamental solution for the Mindlin plate theory with damping is derived in the Laplace transform domain first time. The applications of this fundamental solution are demonstrated by the method of fundamental solution (MFS). All variables in the time domain can be obtained by the Durbin's Laplace transform inversion method. Numerical examples demonstrate the accuracy of the method of fundamental solution and comparisons have been made with analytical solutions. To model the cutting machining process, a moving concentrated force on the plate has been investigated. The proposed MFS is shown to be simple to implement and gives satisfactory results for the shear deformable plate under dynamic loads with damping.
\end{abstract}

Key words: Reissner/Mindlin plate, fundamental solution, Laplace transformation, boundary element method, method of fundamental solution, cutting forces.

*Correspondence: Email: p.h.wen@qmul.ac.uk, Tel: 44207882 5371, Fax: 442089831007 


\section{Introduction}

In metal cutting, the effects of the cutting forces are extremely important as this can mean a difference between successful/unsuccessful machining. The cutting forces give rise to the dynamic response experienced in both the workpiece and tool/machine. This response can either be stable or unstable (regenerative severe vibration known as chatter). Surface dimensional error is also induced by the deflection of the workpiece or tool. In the interest of achieving higher material removal rates, optimum cutting conditions are required. It is due to this reason that there have been quite a number of studies in the literature into the response of the workpiece and tool to the cutting forces.

The cutting force system experience in milling is highly nonlinear and complex. A range of models both analytical and numerical modes have been proposed in the literature to predict the cutting forces, some of which are sometimes tool and workpiece geometry and material dependent. One of the most favoured approaches to predicting the cutting force in milling is to assume the force is proportional to the uncut chip thickness (see Tlusty and MacNeil [1]). Altintas and Engin [2] gave a general definition of the model for different tools and Gradisek et al. [3] furthered his work. The proportionality constants are known as the cutting force coefficients. These are either obtained through Merchant force definitions for (as shown by Altintas [4]) or through mechanistic approach (Gradisek et al. [3]). Either of these methods have lead to very good predictions of the cutting forces acting on the tool/workpiece (Altintas [4]).

To model plate structures, we have two main theories: classical thin plate theory (Kirchhoff's theory) and the shear deformable plate theory (Mindlin-Reissner's theory). Numerical methods to solve plate problems, such as the finite element method and boundary element method, have been well developed since the past decades for both thin and thick plate problems. Apparently, the Kichhoff's theory ignores the effect of shear deformation through the thickness and therefore the elasticity waves are not able to be captured accurately. Ratchev et al. [5] proposed a finite element to model the error in machining of thin wall through the deflection induced by the cutting forces. The finite element is used to obtain the dynamic response of the workpiece during cutting. Tsai and Liao [6] also developed a finite element model to predict the dimensional error induced by the cutting force model. The finite element method was also used by Bravo et al. [7] to obtain the dynamic properties of 
the workpiece for different modes and these properties were used to obtain the dynamic response to the cutting force.

The boundary integral formulation was introduced to reduce the dimensionality of a problem by Simogliana in elasticity theory (see the most comprehensive review of the boundary element method by Cheng et al [8]). For shear deformable Reissner/Mindlin's plates, the boundary integral equation method was reported by Vander Weeën [9] for static problems. Ante et al $[10,11]$ firstly derived the fundamental solution for Reissner plate in the frequency domain and demonstrated its application for dynamic problems. Later, the direct boundary element formulation for Reissner/Mindlin's plate bending under dynamic loads was presented by Wen et al [12], in which the fundamental solutions of displacement and traction in the Laplace transform domain were derived using displacement potential functions. Recently, the fundamental solutions for a Mindlin plate resting on an elastic foundation were derived by Wen [13] and the method of fundamental solution was demonstrated. In their work, the behaviour of the three flexural waves, i.e. slow flexural, fast flexural and thickness shear waves, was studied comprehensively. Chen et al [14] derived the general and the fundamental solutions of varied orders of thin plate, Berger plate, and Winkler plate. These solutions can be used for the multiple reciprocity BEM, dual reciprocity BEM etc.

In recent years, there has been a growing interest in meshless method for the numerical solutions, see Atluri [15]. The method of fundamental solution is regarded as one of the mesh free methods since MFS does not require an elaborate discretisation of the boundary. The integrations over the boundary and domain are avoided successfully and the solutions in the interior of the domain are evaluated without extra treatments. The main idea of the MFS consists of approximating solutions by linear combination of fundamental solutions with respect to the source points, which are located outside the domain. This method was originally introduced by Kupradze et al. [16] and was successfully applied for solving a wide range of boundary value problems, when the fundamental solution is available for the governing partial deferential equations. The method of fundamental solution which was applied firstly to the Kirchhoff plate on a Winkler's foundation was found by Wen [17-18]. A comprehensive review of MFS for two and three dimensions elasticity was given by Marin et al. [19] and for diffusion equations by Chen et al. [20]. 
The effect of impact dynamics in milling of thin-walled components was investigated both experimentally and numerically by Davies et al. [21]. An approximate technique called single-mode Galerkin projection was employed to obtain an ordinary differential equation that describes the workpiece dynamics. However, the effect of elasticity waves traveling in the plate can not be captured by using Kirchhoff plate theory. Further more, the influence of the movement of the cutter on the plate surface should be taken into account particularly for high speed machining, which makes the numerical modeling more difficult. To investigate elasticity waves due to cutting forces with higher accuracy, Reissner/Mindlin's plate theory needs to be introduced as discussed in details by Wen et al. [12]. Considering the shortage of analytical and numerical investigation for the moving forces, this paper deduced the fundamental solution in the Laplace domain for moderate thick plate (Reisnner/Mindlin's theory) with damping. These fundamental solutions can be used to derive the boundary element formulations directly. However, the applications of this fundamental solution in this paper are demonstrated only by MFS, one of the mesh free techniques, for a dynamic cutting force moving on the plate surface. The response due to a moving cutting force has shown to be significant. Comparisons are made with analytical solutions for a simply supported plate to demonstrate the accuracy and convergence of MFS. It is well known that there are still problems of stability and convergence for the method of fundamental solution and the inversion of the Laplace transformation too. However, we are still able to obtain stable solutions in the large range selections of free parameter particularly for the elasticity wave propagations. There are a large number of articles dealing with the stability of the MFS and the inversion techniques of the Laplace transformation.

\section{Fundamental solution for Mindlin's plate with damping}

With the small motion assumption, the governing equations for deflection and rotations for moderate thick with dynamic load and damping force can be expressed, see Wen et al. [12], as

$$
\begin{aligned}
& \frac{D}{2}\left[(1-v) \nabla^{2} w_{\alpha}+(1+v) w_{\beta, \beta \alpha}\right]-\kappa \mu h\left(w_{3, \alpha}+w_{\alpha}\right)=\frac{\rho h^{3}}{12} \frac{\partial^{2} w_{\alpha}}{\partial t^{2}} \\
& \kappa \mu h\left(\nabla^{2} w_{3}+w_{\alpha, \alpha}\right)-c \frac{\partial w_{3}}{\partial t}+q(t)=\rho h \frac{\partial^{2} w_{3}}{\partial t^{2}}
\end{aligned}
$$


where $q(t)$ is applied load in the domain and $w_{\alpha}$ (see Figure 1) denotes rotations with respecting to the axis $x_{\alpha}$ and $w_{3}$ is the out-of-plane deflection, where Greek indices vary from 1 to 2 . Parameter $\kappa$ denotes the shear coefficient $\left(\kappa=\pi^{2} / 12\right.$ for Mindlin's theory and $\kappa=5 / 6$ for Reissner's theory respectively), the bending stiffness of the plate $D=E h^{3} / 12\left(1-v^{2}\right)$, the shear modulus $\mu=E / 2(1+v)$ and $h$ denotes the thickness of the plate, $\rho$ is the density of the plate, $E$ and $v$ are elastic constants and $c$ is the damping coefficient. The components of moment $M_{\alpha \beta}$ and shear forces $Q_{\alpha}$ can be written, in terms of displacements, as

$$
\begin{aligned}
& M_{\alpha \beta}=\frac{1-v}{2} D\left(w_{\alpha, \beta}+w_{\beta, \alpha}+\frac{2 v}{1-v} w_{\gamma, \gamma} \delta_{\alpha \beta}\right) \\
& Q_{\alpha}=\kappa \mu h\left(w_{\alpha}+w_{3, \alpha}\right)
\end{aligned}
$$

in which $\delta_{\alpha \beta}$ denotes the Kronecker delta function. Considering the Laplace transform of a function $f(\mathbf{x}, t)$

$$
L[f(\mathbf{x}, t)]=\tilde{f}(\mathbf{x}, p)=\int_{0}^{\infty} f(\mathbf{x}, t) e^{-p t} d t
$$

where $p$ is the parameter of the Laplace transformation. Applying the Laplace transform to the governing equations (1) results

$$
\begin{aligned}
& 6 D\left\lfloor(1-v) \nabla^{2} \tilde{w}_{\alpha}+(1+v) \tilde{w}_{\beta, \beta \alpha}\right\rfloor-12 \kappa \mu h\left(\tilde{w}_{3, \alpha}+\tilde{w}_{\alpha}\right)=\rho h^{3} p^{2} \tilde{w}_{\alpha} \\
& \kappa \mu h\left(\nabla^{2} \tilde{w}_{3}+\tilde{w}_{\alpha, \alpha}\right)-c p \tilde{w}_{3}+\tilde{q}=\rho h p^{2} \tilde{w}_{3}
\end{aligned}
$$

It is apparent that the rotations and the deflection can be written in terms of three displacement potentials $\phi_{k}(k=1,2,3)$, see Sih et al. [22] and Wen et al. [13], as

$$
\begin{aligned}
& \tilde{w}_{1}=\left(\xi_{1}-1\right) \phi_{1,1}+\left(\xi_{2}-1\right) \phi_{2,1}+\phi_{3,2} \\
& \tilde{w}_{2}=\left(\xi_{1}-1\right) \phi_{1,2}+\left(\xi_{2}-1\right) \phi_{2,2}+\phi_{3,1} \\
& \tilde{w}_{3}=\phi_{1}+\phi_{2}
\end{aligned}
$$

where non-dimensional parameters $\xi_{\beta}$ are two roots in the following equation, for the Mindlin's plate (i.e. $\kappa=\pi^{2} / 12$ ), as 


$$
\begin{aligned}
& {\left[1+\left(\frac{p}{\omega_{0}}\right)^{2}\right] \xi^{2}-\frac{p}{\omega_{0}}\left\{\frac{p}{\omega_{0}}+\frac{24}{(1-v) \pi^{2}}\left[\bar{c}+\frac{p}{\omega_{0}}\right]\right\}} \\
& +\frac{24}{(1-v) \pi^{2}} \frac{p}{\omega_{0}}\left[\bar{c}+\frac{p}{\omega_{0}}\right]=0
\end{aligned}
$$

where $\omega_{0}$ denotes the cut-off frequency $\pi c_{2} / h, c_{2}=\sqrt{\mu / \rho}$ is the velocity of shear wave and non-dimensional damping coefficient $\bar{c}=c_{2} c / \pi \mu$. As in the Laplace domain, the parameter $p$ is complex, thus the two roots of the above equation $\xi_{\beta}$ are complex values in general. Substituting (5) into (4) gives the Helmholz type differential equations for displacement potentials as the following

$$
\left(\nabla^{2}-\alpha_{k}^{2}\right) \phi_{k}=0 \quad k=1,2,3
$$

where

$$
\alpha_{\beta}^{2}=\frac{12}{\xi_{\beta} h^{2}} \frac{p}{\omega_{0}}\left[\bar{c}+\frac{p}{\omega_{0}}\right], \quad \beta=1,2
$$

and

$$
\alpha_{3}^{2}=\frac{\pi^{2}}{h^{2}}\left[1+\left(\frac{p}{\omega_{0}}\right)^{2}\right]
$$

For a symmetric problem with respecting to the axis $x_{1}$, the general solutions of the above equations can be obtained (see Wen et al. [12] for plate bending) by Fourier transformation as

$$
\begin{aligned}
& \phi_{1}=\frac{2}{\pi} \int_{0}^{\infty} \mathrm{A}_{1}(s) e^{-\beta_{1} x_{2}} \cos \left(s x_{1}\right) d s \\
& \phi_{2}=\frac{2}{\pi} \int_{0}^{\infty} \mathrm{A}_{2}(s) e^{-\beta_{2} x_{2}} \cos \left(s x_{1}\right) d s
\end{aligned}
$$

$$
\phi_{3}=\frac{2}{\pi} \int_{0}^{\infty} \mathrm{A}_{3}(s) e^{-\beta_{1} x_{2}} \sin \left(s x_{1}\right) d s
$$

where $\beta_{k}^{2}=s^{2}+\alpha_{k}^{2}$ and $A_{k}(s)$ are unknown coefficients which are to be determined by boundary conditions for each point force. Therefore, we have the rotations and the deflection as follows 


$$
\begin{aligned}
& \tilde{w}_{1}=\frac{2}{\pi} \int_{0}^{\infty}\left[\left(1-\xi_{1}\right) s A_{1}(s) e^{-\beta_{1} x_{2}}+\left(1-\xi_{2}\right) s A_{2}(s) e^{-\beta_{2} x_{2}}-\beta_{3} A_{3}(s) e^{-\beta_{3} x_{2}}\right] \sin \left(s x_{1}\right) d s \\
& \tilde{w}_{2}=\frac{2}{\pi} \int_{0}^{\infty}\left[\left(1-\xi_{1}\right) \beta_{1} A_{1}(s) e^{-\beta_{1} x_{2}}+\left(1-\xi_{2}\right) \beta_{1} A_{2}(s) e^{-\beta_{2} x_{2}}-s A_{3}(s) e^{-\beta_{3} x_{2}}\right] \cos \left(s x_{1}\right) d s \\
& \tilde{w}_{3}=\frac{2}{\pi} \int_{0}^{\infty}\left[A_{1}(s) e^{-\beta_{1} x_{2}}+A_{2}(s) e^{-\beta_{2} x_{2}}\right] \cos \left(s x_{1}\right) d s \quad x_{2} \geq 0
\end{aligned}
$$

The components of moment $\tilde{M}_{\alpha \beta}$ and shear forces $\tilde{Q}_{\alpha}$ in transformed domain can then be obtained as

$$
\begin{gathered}
\tilde{M}_{11}=\frac{2 D}{\pi} \int_{0}^{\infty}\left[\left(1-\xi_{1}\right)\left(s^{2}-v \beta_{1}^{2}\right) A_{1}(s) e^{-\beta_{1} x_{2}}+\left(1-\xi_{2}\right)\left(s^{2}-v \beta_{2}^{2}\right) A_{2}(s) e^{-\beta_{2} x_{2}}\right. \\
\left.\quad-s \beta_{3}(1-v) A_{3}(s) e^{-\beta_{3} x_{2}}\right] \cos \left(s x_{1}\right) d s \\
\tilde{M}_{22}=\frac{2 D}{\pi} \int_{0}^{\infty}\left[\left(1-\xi_{1}\right)\left(v s^{2}-\beta_{1}^{2}\right) A_{1}(s) e^{-\beta_{1} x_{2}}+\left(1-\xi_{2}\right)\left(v s^{2}-\beta_{2}^{2}\right) A_{2}(s) e^{-\beta_{2} x_{2}}\right. \\
\left.\quad+s \beta_{3}(1-v) A_{3}(s) e^{-\beta_{3} x_{2}}\right] \cos \left(s x_{1}\right) d s \\
\tilde{M}_{12}=-\frac{(1-v) D^{\infty}}{\pi} \int_{0}^{\infty}\left[2 s \beta_{1}\left(1-\xi_{1}\right) A_{1}(s) e^{-\beta_{1} x_{2}}+2 s \beta_{2}\left(1-\xi_{2}\right) A_{2}(s) e^{-\beta_{2} x_{2}}\right. \\
\left.+\left(\beta_{3}^{2}+s^{2}\right) A_{3}(s) e^{-\beta_{3} x_{2}}\right] \sin \left(s x_{1}\right) d s
\end{gathered}
$$

and

$$
\begin{aligned}
& \tilde{Q}_{1}=-\frac{2 \kappa \mu h}{\pi} \int_{0}^{\infty}\left[s \xi_{1} A_{1}(s) e^{-\beta_{1} x_{2}}+s \xi_{2} A_{2}(s) e^{-\beta_{2} x_{2}}+\beta_{3} A_{3}(s) e^{-\beta_{3} x_{2}}\right] \sin \left(s x_{1}\right) d s \\
& \tilde{Q}_{2}=-\frac{2 \kappa \mu h}{\pi} \int_{0}^{\infty}\left[\beta_{1} \xi_{1} A_{1}(s) e^{-\beta_{1} x_{2}}+\beta_{2} \xi_{2} A_{2}(s) e^{-\beta_{2} x_{2}}+s A_{3}(s) e^{-\beta_{3} x_{2}}\right] \cos \left(s x_{1}\right) d s
\end{aligned}
$$

For example, considering a quarter of infinite domain and one point moment acting at the origin, three coefficients $A_{k}(s)$ are obtained

$$
A_{1}(s)=-\frac{1}{4 D\left(\alpha_{2}^{2}-\alpha_{1}^{2}\right)}, A_{2}(s)=\frac{1}{4 D\left(\alpha_{2}^{2}-\alpha_{1}^{2}\right)}, A_{3}(s)=\frac{s\left(\xi_{2}-\xi_{1}\right)}{4 D\left(\alpha_{2}^{2}-\alpha_{1}^{2}\right) \beta_{3}}
$$

Consider the relationship of integrals with the zero-order modified Bessel function

$$
K_{0}\left(\alpha_{j} r\right)=\int_{0}^{\infty} \frac{\cos \left(s x_{1}\right)}{\sqrt{s^{2}+\alpha_{j}^{2}}} e^{-\sqrt{s^{2}+\alpha_{j}^{2}} x_{2}} d s
$$


in which $K_{0}\left(z_{1}\right)$ represents the zero order modified Bessel function and $r=\sqrt{x_{1}^{2}+x_{2}^{2}}$. The general form of fundamental solutions for rotations and deflection $\tilde{U}_{i k}$ in the Laplace domain for three unit concentrated force (point forces) can be written as

$$
\begin{aligned}
& \tilde{U}_{\alpha \beta}(\mathbf{x}, p)=\frac{1}{\pi(1-v) D \alpha_{3}^{2}\left(\xi_{2}-\xi_{1}\right)}\left(f_{, \alpha \beta}+g \delta_{\alpha \beta}\right) \\
& \tilde{U}_{\alpha 3}(\mathbf{x}, p)=\frac{1}{\pi(1-v) D \alpha_{3}^{2}\left(\xi_{2}-\xi_{1}\right)} h_{, \alpha}
\end{aligned}
$$

and

$$
\begin{aligned}
& \tilde{U}_{3 \alpha}(\mathbf{x}, p)=\frac{\left(1-\xi_{2}\right)\left(1-\xi_{1}\right)}{2 \pi \kappa \mu h\left(\xi_{2}-\xi_{1}\right)} h_{, \alpha} \\
& \tilde{U}_{33}(\mathbf{x}, p)=-\frac{1}{2 \pi \kappa \mu\left(\xi_{2}-\xi_{1}\right)}\left[\left(1-\xi_{2}\right) K_{0}\left(z_{1}\right)-\left(1-\xi_{1}\right) K_{0}\left(z_{2}\right)\right]
\end{aligned}
$$

where $z_{j}=\alpha_{j} r$ and non-dimensional functions

$$
\begin{aligned}
& f=\left(1-\xi_{1}\right) K_{0}\left(z_{1}\right)-\left(1-\xi_{2}\right) K_{0}\left(z_{2}\right)-\left(\xi_{2}-\xi_{1}\right) K_{0}\left(z_{3}\right) \\
& \mathrm{g}=\left(\xi_{2}-\xi_{1}\right) \alpha_{3}^{2} K_{0}\left(\mathrm{z}_{3}\right) \\
& h=K_{0}\left(z_{1}\right)-K_{0}\left(z_{2}\right)
\end{aligned}
$$

By the use of the property of modified Bessel functions, the fundamental solutions for the displacement fundamental solutions can be rewritten as

$$
\begin{aligned}
& \tilde{U}_{\alpha \beta}=\frac{1}{\pi D(1-v) \alpha_{3}^{2}\left(\xi_{2}-\xi_{1}\right)}\left[\left(1-\xi_{1}\right) \alpha_{1} K_{1}\left(z_{1}\right)-\left(1-\xi_{2}\right) \alpha_{2} K_{1}\left(z_{2}\right)-\left(\xi_{2}-\xi_{1}\right) \alpha_{3} K_{1}\left(z_{3}\right)\right) \\
& \left(2 r_{, \alpha} r_{, \beta}-\delta_{\alpha \beta}\right) / r+\left(\left(1-\xi_{1}\right) \alpha_{1}^{2} K_{0}\left(z_{1}\right)-\left(1-\xi_{2}\right) \alpha_{2}^{2} K_{0}\left(z_{2}\right)\right. \\
& \left.\left.-\left(\xi_{2}-\xi_{1}\right) \alpha_{3}^{2} K_{0}\left(z_{3}\right)\right) r_{, \alpha} r_{, \beta}+\left(\xi_{2}-\xi_{1}\right) \alpha_{3}^{2} K_{0}\left(z_{3}\right) \delta_{\alpha \beta}\right] \\
& \tilde{U}_{\alpha 3}=\frac{1}{\pi D(1-v) \alpha_{3}^{2}\left(\xi_{2}-\xi_{1}\right)}\left[\alpha_{1} K_{1}\left(z_{1}\right)-\alpha_{2} K_{1}\left(z_{2}\right)\right] r_{, \alpha} \\
& \tilde{U}_{3 \alpha}=-\frac{\left(1-\xi_{2}\right)\left(1-\xi_{1}\right)}{2 \pi \kappa \mu h\left(\xi_{2}-\xi_{1}\right)}\left[\alpha_{1} K_{1}\left(z_{1}\right)-\alpha_{2} K_{1}\left(z_{2}\right)\right] r_{, \alpha} \\
& \tilde{U}_{33}=-\frac{\left(1-\xi_{2}\right)\left(1-\xi_{1}\right)}{2 \pi \kappa \mu h\left(\xi_{2}-\xi_{1}\right)}\left[\frac{K_{0}\left(z_{1}\right)}{\left(1-\xi_{1}\right)}-\frac{K_{0}\left(z_{2}\right)}{\left(1-\xi_{2}\right)}\right]
\end{aligned}
$$

Then, the traction fundamental solutions are obtained from the relationships in (2) 


$$
\begin{aligned}
& \tilde{T}_{\alpha \beta}=\frac{1}{2 \pi \alpha_{3}^{2}\left(\xi_{2}-\xi_{1}\right)}\left[-\left\{\left(1-\xi_{1}\right) \alpha_{1}\left[2 K_{1}\left(z_{1}\right)+z_{1} K_{0}\left(z_{1}\right)\right]-\left(1-\xi_{2}\right) \alpha_{2}\left[2 K_{1}\left(z_{1}\right)+\right.\right.\right. \\
& \left.\left.z_{1} K_{0}\left(z_{1}\right)\right]-\left(\xi_{2}-\xi_{1}\right) \alpha_{3}\left[2 K_{1}\left(z_{3}\right)+z_{3} K_{0}\left(z_{3}\right)\right]\right\} \times \\
& \frac{2}{r^{2}}\left[4 r_{, \alpha} r_{, \beta} r_{, n}-\left(r_{, \alpha} n_{\beta}+r_{, \beta} n_{\alpha}+r_{, n} \delta_{\alpha \beta}\right)\right]-2\left[\left(1-\xi_{1}\right) \alpha_{1}^{3} K_{1}\left(z_{1}\right)-\right. \\
& \left.\left(1-\xi_{2}\right) \alpha_{2}^{3} K_{1}\left(z_{2}\right)-\left(\xi_{2}-\xi_{1}\right) \alpha_{3}^{3} K_{1}\left(z_{3}\right)\right] r_{, \alpha} r_{, \beta} r_{, n}-\left(\xi_{2}-\xi_{1}\right) \alpha_{3}^{3} K_{1}\left(z_{3}\right)\left(r_{, \beta} n_{\alpha}+r_{, n} \delta_{\alpha \beta}\right) \\
& \left.+\frac{2 v}{1-v}\left(-\left(1-\xi_{1}\right) \alpha_{1}^{3} K_{1}\left(z_{1}\right)+\left(1-\xi_{2}\right) \alpha_{2}^{3} K_{1}\left(z_{2}\right)\right) r_{, \alpha} n_{\beta}\right] \\
& T_{\alpha 3}=-\frac{\kappa \mu h}{\pi D(1-v) \alpha_{3}^{2}\left(\xi_{2}-\xi_{1}\right)}\left[\left\{\xi_{1} \alpha_{1} K_{1}\left(z_{1}\right)-\xi_{2} \alpha_{2} K_{1}\left(z_{2}\right)+\left(\xi_{2}-\xi_{1}\right) \alpha_{3} K_{1}\left(z_{3}\right)\right\} \times\right. \\
& \frac{1}{r}\left(2 r_{, \alpha} r_{, n}-n_{\alpha}\right)+\left\{\xi_{1} \alpha_{1}^{2} K_{0}\left(z_{1}\right)-\xi_{2} \alpha_{2}^{2} K_{0}\left(z_{2}\right)+\right. \\
& \left.\left.\left(\xi_{2}-\xi_{1}\right) \alpha_{3}^{2} K_{0}\left(z_{3}\right)\right\} r_{, \alpha} r_{, n}-\left(\xi_{2}-\xi_{1}\right) \alpha_{3}^{2} K_{0}\left(z_{3}\right) n_{\alpha}\right] \\
& \tilde{T}_{3 \alpha}=\frac{(1-v)\left(1-\xi_{2}\right)\left(1-\xi_{1}\right) D}{2 \pi \kappa \mu h\left(\xi_{2}-\xi_{1}\right)}\left[\left\{\alpha_{1} K_{1}\left(z_{1}\right)-\alpha_{2} K_{1}\left(z_{2}\right)\right\} \frac{1}{r}\left(2 r_{, \alpha} r_{, n}-n_{\alpha}\right)\right. \\
& \left.+\left\{\alpha_{1}^{2} K_{0}\left(z_{1}\right)-\alpha_{2}^{2} K_{0}\left(z_{2}\right)\right\} r_{, \alpha} r_{, n}+\frac{v}{1-v}\left\{\alpha_{1}^{2} K_{0}\left(z_{1}\right)-\alpha_{2}^{2} K_{0}\left(z_{2}\right)\right\} n_{\alpha}\right] \\
& T_{33}=\frac{\left(1-\xi_{2}\right)\left(1-\xi_{1}\right)}{2 \pi\left(\xi_{2}-\xi_{1}\right)}\left[\frac{\alpha_{1} K_{1}\left(z_{1}\right)}{1-\xi_{1}}-\frac{\alpha_{2} K_{1}\left(z_{2}\right)}{1-\xi_{2}}-\left\{\alpha_{1} K_{1}\left(z_{1}\right)-\alpha_{2} K_{1}\left(z_{2}\right)\right\}\right] r_{, n}
\end{aligned}
$$

where $n_{\beta}$ denotes the component of the outward normal vector to the boundary of the plate (Г) and $r_{, n}=r_{, \alpha} n_{\alpha}$.

\section{Method of fundamental solution in Laplace domain}

In the MFS, we distribute source points outside the physical domain in order to avoid the singularities of fundamental solution. By using the principle of superposition for linear elasticity, Wen [17-18] demonstrated the MFS for Kirchhoff plate resting on an elastic foundation subjected to static loads. As the superposition principle is still valid in the transformed domain, the approximate solutions of the displacement (deflection and rotations) and the resultants of moment and shear force in the Laplace domain at the boundary collocation point can be expressed as 


$$
\begin{aligned}
& \tilde{w}_{i}(\mathbf{P})=\tilde{w}_{i}^{*}(\mathbf{P})+\sum_{n=1}^{N} \tilde{U}_{i k}(\mathbf{P}, \mathbf{Q}) c_{k}^{n}(\mathbf{Q}) \\
& \tilde{p}_{i}(\mathbf{P})=\tilde{p}_{i}^{*}(\mathbf{P})+\sum_{n=1}^{N} \tilde{T}_{i k}(\mathbf{P}, \mathbf{Q}) c_{k}^{n}(\mathbf{Q}) \quad \mathbf{P} \in \Gamma, \quad i=1,2,3
\end{aligned}
$$

where $\tilde{p}_{\alpha}=\tilde{M}_{\alpha \beta} n_{\beta}, \tilde{p}_{3}=\tilde{Q}_{\beta} n_{\beta}, \quad \tilde{w}_{i}^{*}(\mathbf{P})$ and $\tilde{p}_{i}^{*}(\mathbf{P})$ present the particular solutions in governing equation (4), $\mathbf{P}$ and $\mathbf{Q}$ denote the collocation and the source points shown in Figure 2 respectively and $c_{k}^{n}$ the unknown densities of concentrated forces at source point $n$. In general, the number of collocation point on the real boundary $N_{c}$ is different from the number of source point outside of domain $N_{s}$. The least square method needs to be utilized to determine all unknown densities if $N_{c}>N_{s}$. In this paper, the case $N_{c}=N_{s}$ is considered only.

In general, the applied load on the plate surface is a function of coordinate. However, if uniform and linear distributed loads are considered in the domain, i.e.

$$
q(\mathbf{P}, t)=q_{0}(t)+q_{1}(t) x_{1}+q_{2}(t) x_{2}
$$

where $q_{0}(t), q_{1}(t)$ and $q_{2}(t)$ are time dependent functions only, we are able to obtain the particular solutions in the Laplace domain analytically as

$\tilde{q}(\mathbf{P}, p)=\tilde{q}_{0}(p)+\tilde{q}_{1}(p) x_{1}+\tilde{q}_{2}(p) x_{2}$

where particular solutions of displacement can be obtained from the governing equation (4) and are given by

$$
\begin{aligned}
& \tilde{w}_{1}^{*}=-\frac{\kappa \mu \tilde{q}_{1}}{p\left(\kappa \mu+\rho h^{2} p^{2}\right)(c+\rho h p)}, \tilde{w}_{2}^{*}=-\frac{\kappa \mu \tilde{q}_{2}}{p\left(\kappa \mu+\rho h^{2} p^{2}\right)(c+\rho h p)}, \\
& \tilde{w}_{3}^{*}=\frac{1}{p(c+\rho h p)}\left[\tilde{q}_{0}(t)+\tilde{q}_{1}(t) x_{1}+\tilde{q}_{2}(t) x_{2}\right]
\end{aligned}
$$

and the particular solutions for the moment and the shear forces are

$$
\begin{aligned}
& \tilde{M}_{11}^{*}=\tilde{M}_{12}^{*}=\tilde{M}_{22}^{*}=0 \\
& \tilde{Q}_{1}^{*}=\frac{\kappa \mu h \tilde{q}_{1}}{p(c+\rho h p)}\left(1-\frac{\kappa \mu}{\kappa \mu+\rho h^{2} p^{2}}\right) \\
& \tilde{Q}_{2}^{*}=\frac{\kappa \mu h \tilde{q}_{2}}{p(c+\rho h p)}\left(1-\frac{\kappa \mu}{\kappa \mu+\rho h^{2} p^{2}}\right)
\end{aligned}
$$


Considering the displacement and traction boundary conditions of the plate, we have following linear algebraic equations:

$$
\begin{array}{ll}
\sum_{n=1}^{N} \tilde{U}_{i k}(\mathbf{P}, \mathbf{Q}) c_{k}^{n}(\mathbf{Q})=\tilde{\bar{w}}_{i}(\mathbf{P})-\tilde{w}_{i}^{*}(\mathbf{P}) \quad \text { for } \quad \mathbf{P} \in \Gamma_{u} \\
\sum_{n=1}^{N} \tilde{T}_{i k}(\mathbf{P}, \mathbf{Q}) c_{k}^{n}(\mathbf{Q})=\tilde{\bar{p}}_{i}(\mathbf{P})-\tilde{p}_{i}^{*}(\mathbf{P}) & \text { for } \quad \mathbf{P} \in \Gamma_{\sigma}
\end{array}
$$

where $\tilde{\bar{w}}_{i}$ and $\tilde{\bar{p}}_{i}$ are specified displacement and traction boundary values in the Laplace domain respectively, $\Gamma_{u}$ and $\Gamma_{\sigma}$ present the displacement and the traction boundaries $\left(\Gamma=\Gamma_{u}+\Gamma_{\sigma}\right)$. Thus a set of unknown intensity of point force can be obtained by solving the linear system in (32) in the Laplace domain for each Laplace parameter $p_{k}, k=0,1,2 \ldots L$. Although the MFS of linear algebraic equation is ill-conditioned, the stable and convergent solutions can be obtained by selecting a proper gap between collocation and source points. The accuracy and sensitivity respecting to the gap were examined by Wen et al. in the static state.

In the Laplace domain, a total number of samples $(L+1)$ in the transformation space $p_{k}$ are selected. The transformed variables are evaluated for these specified transform parameters. Then the variables in time domain can be determined by the Laplace inversion technique. Here, the method proposed by Durbin [24] is adopted. Demonstration of the Durbin's Laplace transform inverse method was made by Wen et al. [25] for the elasticity wave propagations for two-dimensional problems. The formula of inversion is written as

$$
f(t)=\frac{2 e^{\eta t}}{T}\left[-\frac{1}{2} \tilde{f}(\eta)+\sum_{k=0}^{L} \operatorname{Re}\left\{\tilde{f}(\eta+2 k \pi i / T) e^{2 k \pi t i / T}\right\}\right]
$$

where $\tilde{f}\left(p_{k}\right)$ denotes the transformed variable in the Laplace domain, the parameter of the Laplace transform $p_{k}=\eta+2 k \pi i / T \quad(i=\sqrt{-1})$. The selection of two free parameters $\eta$ and $T$ affects the accuracy of inversion slightly.

\section{Response of moving point forces on surface of infinite plate}

In the modern milling machining technology, high-speed milling processes can produce more accurate and repeatable results and reduce the costs of assembly and fixture storage by allowing for several separate components to be combined into a single, monolithic 
machined part. Because of high-spindle speeds and high-slide accelerations, maintaining accuracy in high-speed machining requires the development of more accurate dynamic models of machines and cutting processes. Therefore, the effect of the impact of load motion with speed should be taken into account in the numerical modeling. Suppose a concentrated force $P$ moving from origin $(0,0)$ to $(b, 0)$ with speed $V$, shown in Figure 3 , the point force can be described as

$$
\begin{array}{ll}
q\left(x_{1}, 0\right)=P \delta\left(x_{1}-V t\right) & 0 \leq x_{1} \leq b \\
q\left(x_{1}, 0\right)=0 & x_{1}<0 \text { or } x_{1}>b
\end{array}
$$

Applying Laplace transformation over moving load above gives

$$
\tilde{q}\left(x_{1}, 0\right)=\frac{P}{V} e^{-p x_{1} / V}\left(H\left(x_{1}\right)-H\left(x_{1}-b\right)\right)
$$

where $H(t)$ denotes Heaviside function. By the principle of superposition, the resultants of moment and shear force at location $\left(x_{1}, x_{2}\right)$ can be written, in an integral form, as

$$
\begin{aligned}
& \tilde{M}_{22}\left(x_{1}, x_{2}\right)=\int_{0}^{b} \tilde{T}_{23}\left(x_{1}-\sigma, x_{2}\right) \tilde{q}(\sigma, 0) d \sigma \\
& \tilde{Q}_{2}\left(x_{1}, x_{2}\right)=\int_{0}^{b} \widetilde{T}_{33}\left(x_{1}-\sigma, x_{2}\right) \tilde{q}(\sigma, 0) d \sigma
\end{aligned}
$$

in which the components of normal are $n_{1}=0, n_{2}=1$. Two observation points are selected as $\mathrm{A}(10 a, a)$ and $\mathrm{B}(10 a, 0.1 a)$, and the length for the moving force $b$ is chosen as $20 a$, where $a$ is the unit of length. Poisson's ratio is taken as 0.3 , thickness $h=0.1 a$ and the two free parameters $\eta=5 / t_{0}$ and $T=80 t_{0}$, where $t_{0}=a / c_{2}$ (unit of time). The total number of samples in the Laplace domain $L=100$ and the damping coefficient $c=0$. Three cases for different point force speed, i.e. $V=0.2 c_{2}, 0.5 c_{2}$ and $c_{2}$, are investigated respective. Figures 4 , 5 and 6 show the variations of moment $M_{22}(t) / P$ and shear force $Q_{2}(t) a / P$ at two observation points against the normalised time $c_{2} t / a$. As the integrals in (36) are regular, the Gaussian integral scheme is employed directly and the relative error of criterion is selected to be $10^{-6}$. The following conclusions can be found from investigation:

(1) before the arrival of elasticity waves (fast wave), the moment and shear force are zero as expected; 
(2) the maximum values both for the moment and shear force occur about the time $t=10 \mathrm{a} / \mathrm{V}$, i.e. when the point force moves to the nearest point of the observation points;

(3) the maximum values both for the moment and shear force increase with increasing of moving speed of the point force;

(4) the results for the moment and shear force are smooth against the normalised time for the lower speed of $V$ and oscillation with high frequency occurs for high speed of $V$;

(5) the moment and shear force decline to zero after time $t>20 a / V$, i.e. whilst the point force disappears.

In addition, the response of a moving concentrated moment (due to the distance between the middle plane and surface for a tangential cutting force along feeding direction) can be obtained in the same way. The moment and shear force in the panel are

$$
\begin{aligned}
& \tilde{M}_{22}\left(x_{1}, x_{2}\right)=\int_{0}^{b} \tilde{T}_{21}\left(x_{1}-\eta, x_{2}\right) \tilde{q}(\eta, 0) d \eta \\
& \tilde{Q}_{2}\left(x_{1}, x_{2}\right)=\int_{0}^{b} \widetilde{T}_{31}\left(x_{1}-\eta, x_{2}\right) \tilde{q}(\eta, 0) d \eta
\end{aligned}
$$

where $q\left(x_{1}, 0\right)=q_{1}=F \delta\left(x_{1}-V t\right) h / 2, F$ is the tangential cutting force (in the time domain).

\section{Numerical examples}

\subsection{A simply supported square plate under uniform load}

In this example, a simply supported square plate of length $a\left(a_{1}=a_{2}\right)$ and thickness $h$ subjected to a uniform static load $q_{0} H(t)$ as shown in Figure 7 is analysed. The aim of this example is to demonstrate the accuracy and the stability of the MFS for a moderate thick plate. Collocation points and source points are distributed uniformly along each edge of the plate, as shown in Figure 8, where $\Delta$ presents the gap between collocation and source points and $d$ is the distance between two collocation points. The solutions are convergent in the region of $2<\Delta / d<10$. More details for the optimal selections of the gap were discussed in [13]. In this example, $\Delta / d=4$. Particular solutions for deflection and rotations under uniform loads are $\tilde{w}_{1}^{*}=0, \quad \tilde{w}_{2}^{*}=0$ and $\tilde{w}_{3}^{*}=\tilde{q}_{0} / p(c+\rho h p)\left(\right.$ where $\left.\tilde{q}_{0}=q_{0} / p\right)$, and all particular solutions for the moment and shear forces are zero. 
Comparison has been made with analytical solution for the Kirchhoff's plate theory. The analytical solution with damping has been deduced in Appendix A. Here Poisson's ratio $v=0.3$ and the thickness of plate $h / a=0.05,0.1$ respectively. Total number of collocation point is taken as 84. Two free parameters for the Laplace inversion method $\eta=5 / t_{0}$ and $T=80 t_{0}$. The variations of the normalised deflection $w_{3} D / q_{0} a^{4}$ at the centre of plate are plotted in Figures $8(\mathrm{a})(\mathrm{b})$ against the normalised $c_{2} t / a$. Here two damping coefficients are considered, i.e. $c=0$ and $c=10 D / c_{2} h a^{2}$. At the beginning of time, the results by Mindlin's and Kirchhoff's plate theories are very close and gradually the gap between them is getting bigger due to the effects of shear deflection. It has been accepted that the solutions by the Mindlin's plate theory is more accurate than that by Kirchhoff's theory. In addition, $t$ t has been found that the similar degree of accuracy can be obtained for the irregular distribution of collocation points. Figures 8(a)(b) show the effect of damping coefficient on the deflection of the plate. With impact load, the plate starts to oscillate about the position of static load and the amplitude of oscillation reduces to zero rapidly as expected.

\subsection{Rectangular plate with three edges built in and the fourth edge free}

In the manufacturing of wing box components, such as ribs and spars, we have many pockets which are separated by thin walls. Therefore, one typical numerical modelling is a rectangular plate $\left(a_{1}=a_{2}=a\right)$ with three edges built in and the fourth edge free as shown in Figure 9. In this example, Poisson's ratio $v=1 / 6$ and $h / a=0.1$. The total number of collocation point is taken as 84 and the number of samples in the Laplace domain $L=100$. Three loading cases are analysed in the following:

\section{(1) Uniform load $q_{0} H(t)$ in the domain}

In order to illustrate the accuracy of this method, finite element method (ABAQUS, v6.6-1) is used in this example. Young's modulus $E=10^{4} \mathrm{~N} / \mathrm{mm}^{2}, v=1 / 6, a=1000 \mathrm{~mm}$, $h=100 \mathrm{~mm}$, density of material $\rho=4.2857 \times 10^{-5} \mathrm{~kg} / \mathrm{mm}^{3}$, damping coefficient $c=0.0857$ $(\mathrm{Ns} / \mathrm{mm})$ and pressure load $q_{0}=1 \mathrm{~N} / \mathrm{mm}^{2}$. In total 2500 quadratic shell elements are used in the finite element modelling. To use the method fundamental solution, the particular solution is the same as that in Example 4.1. The variations of deflection $w_{3}(\mathrm{~mm})$ on the middle top 
edge and centre of the plate are plotted in Figures 10 and 11 against the time $t(\mathrm{~s})$. It is clear that the frequencies of vibration for these two points are the same and the amplitudes of oscillation reduce to zero rapidly with damping effect. As can be seen, the results from the FEM and MFS models are in excellent agreement.

\section{(2) A moving point force}

Assume that there is a constant cutting force $P$ moving from the left hand side to right hand side of the plate (dash line in Figure 7) and the dynamic load $q\left(x_{1}, 0\right)=P \delta\left(x_{1}-V t\right)$, $-0.4 a<x_{1}<0.4 a$. The particular solution can be selected as the solution in section 4 , i.e. a moving point force in an infinite plane. To illustrate the effect of the moving speed of a concentrated force, two different velocities of moving force are applied in this example, i.e. $V / c_{2}=0.1$ and 0.2 respectively. Three points $\mathrm{A}(-0.25 a, 0.5 a), \mathrm{B}(0,0.5 a)$ and $\mathrm{C}(0.25 a, 0.5 a)$ on the top of the plate are observed. The results in Figures 12(a)(b) and 13(a)(b) show the variations of the normalised deflection $w_{3} \mathrm{D} / \mathrm{Pa}^{2}$ against the normalised time $c_{2} t / a$ for the different speeds of moving force. In fact, the maximum deflection of the plate does not change much for different speed of the moving force. Arrival times of the elasticity wave to these observing points can be seen clearly from these figures. Due to the damping effect, the deflection declines to zero rapidly. This behaviour of damping in a plate with three edges built in and one edge free is very important for the cutting analysis of milling process and it can be used to determine the tool moving speed on the work piece. In addition, the maximum deflection can be found, for all cases, on the middle point B as expected. In fact, the cutting force is not constant and is a function of depth of machining. This depth depends on the deflection of the cutting point as well (non-linearity). This non linear dynamic phenomenon is deferred as future work.

\section{Conclusions}

In this paper, the fundamental solution for the Mindlin plates with damping was derived in the Laplace transform domain and the method of fundamental solution was demonstrated. Excellent agreement with analytical solution for a simply supported plate was achieved. As one of the mesh free techniques, the MFS has the most advantages of the mesh free method and demonstrates three major features in their computations: simplicity, 
accuracy and efficiency. We can conclude with the following observations: (1) MFS is suitable for the shear deformable plate for dynamic problems with damping; (2) Constant cutting force moving on the plate surface can be simulated in the Laplace transform domain; (3) MFS is excellent for complex boundary constrain condition; (4) Disadvantages of the MFS are also evident, such as the fundamental solutions must be available for the problem and the optimized source point distribution needs to be investigated. Finally, the boundary integral formulations with these fundamental solutions can be derived directly.

\section{Acknowledgment}

This work has been carried out partly with the support by EPSRC CASE and Airbus UK.

\section{Appendix A}

For the Kirchhoff's plate theory, the governing equation with damping is

$$
D \nabla^{2} \nabla^{2} w-c \frac{\partial w}{\partial t}+q=\rho h \frac{\partial^{2} w}{\partial t^{2}}
$$

where $w$ denotes the deflection of plate. Considering the boundary condition for a simply supported plate, the general solution can be written in the series form as

$$
w=\sum_{m=1}^{\infty} \sum_{n=1}^{\infty} f_{m n}(t) \sin \frac{m \pi x_{1}}{a_{1}} \sin \frac{m \pi x_{2}}{a_{2}}
$$

where $a_{1}$ and $a_{2}$ are width and height of a rectangular plate. Applying the Laplace transform to (1) yields

$$
\tilde{w}=\sum_{m=1}^{\infty} \sum_{n=1}^{\infty} \tilde{f}_{m n}(p) \sin \frac{m \pi x_{1}}{a_{1}} \sin \frac{m \pi x_{2}}{a_{2}}
$$

And the governing equation (1) gives

$$
\tilde{f}_{m n}=\frac{\tilde{q}_{m n}}{\pi^{4} D\left(\frac{m^{2}}{a_{1}^{2}}+\frac{n^{2}}{a_{2}^{2}}\right)+c p+\rho h p^{2}}
$$

where $\tilde{q}_{m n}$ are the coefficients for double Fourier transformation to the applied load in the transform domain and can be obtained by 
$\tilde{q}_{m n}=\frac{4}{\pi^{2}} \int_{0}^{a_{1}} \int_{0}^{a_{2}} \tilde{q}\left(x_{1}, x_{2}\right) \sin \frac{m \pi x_{1}}{a_{1}} \sin \frac{n \pi x_{2}}{a_{2}} d x_{1} d x_{2}$

For a uniformly distributed load, i.e. $q\left(x_{1}, x_{2}\right)=q_{0} H(t)$ then $\tilde{q}\left(x_{1}, x_{2}\right)=q_{0} / p$, we have $\tilde{q}_{m n}=\frac{16 q_{0}}{p m n \pi^{2}}$

and deflection in the Laplace domain becomes

$$
\widetilde{w}=\frac{16 q_{0}}{p \pi^{2}} \sum_{m=1}^{\infty} \sum_{n=1}^{\infty} \frac{1}{m n\left(p-p_{1}\right)\left(p-p_{2}\right)} \sin \frac{m \pi x_{1}}{a_{1}} \sin \frac{m \pi x_{2}}{a_{2}}
$$

where

$$
\begin{aligned}
& p_{1}=\alpha+\beta i, \quad p_{2}=\alpha-\beta i, \quad i=\sqrt{-1} \\
& \alpha=-\frac{\lambda}{2}, \quad \beta=\frac{1}{2} \sqrt{4 \varepsilon-\lambda^{2}}, \quad \lambda=\frac{c_{2}^{2} h^{2} c}{6 D(1-v)}, \quad \varepsilon=\frac{c_{2}^{2} h^{2} \pi^{4}}{6(1-v)}\left(\frac{m^{2}}{a_{1}^{2}}+\frac{n^{2}}{a_{2}^{2}}\right)^{2}
\end{aligned}
$$

It is easy to obtain the solution of deflection in time domain as following

$$
w(t)=\frac{16 q_{0}}{\pi^{6}} \sum_{m=1}^{\infty} \sum_{n=1}^{\infty} \frac{\sin \frac{m \pi x_{1}}{a_{1}} \sin \frac{m \pi x_{2}}{a_{2}}}{m n\left(\frac{m^{2}}{a_{1}^{2}}+\frac{n^{2}}{a_{2}^{2}}\right)^{2}}\left[1+\frac{1}{p_{1}-p_{2}}\left(p_{2} e^{p_{1} t}-p_{1} e^{p_{2} t}\right)\right]
$$

If damping coefficient satisfies

$$
c<\frac{2 \sqrt{6(1-v)} D \pi^{2}}{c_{2} h}\left(\frac{m^{2}}{a_{1}^{2}}+\frac{n^{2}}{a_{2}^{2}}\right)
$$

we can simplify the solution of deflection by

$$
w(t)=\frac{16 q_{0}}{\pi^{6}} \sum_{m=1}^{\infty} \sum_{n=1}^{\infty} \frac{\sin \frac{m \pi x_{1}}{a_{1}} \sin \frac{m \pi x_{2}}{a_{2}}}{m n\left(\frac{m^{2}}{a_{1}^{2}}+\frac{n^{2}}{a_{2}^{2}}\right)^{2}}\left[1+\frac{e^{\alpha t}}{\beta}(\alpha \sin \beta t-\beta \cos \beta t)\right]
$$

Otherwise, the deflection can be presented as

$$
w(t)=\frac{16 q_{0}}{\pi^{6}} \sum_{m=1}^{\infty} \sum_{n=1}^{\infty} \frac{\sin \frac{m \pi x_{1}}{a_{1}} \sin \frac{m \pi x_{2}}{a_{2}}}{m n\left(\frac{m^{2}}{a_{1}^{2}}+\frac{n^{2}}{a_{2}^{2}}\right)^{2}}\left[1+\frac{1}{2 \sqrt{\lambda^{2}-4 \varepsilon}}\left\langle\left(\lambda-\sqrt{\lambda^{2}-4 \varepsilon}\right) e^{-\frac{t}{2}\left(\lambda+\sqrt{\lambda^{2}-4 \varepsilon}\right)}-\right.\right.
$$




$$
\left.\left.\left(\lambda+\sqrt{\lambda^{2}-4 \varepsilon}\right) e^{-\frac{t}{2}\left(\lambda-\sqrt{\lambda^{2}-4 \varepsilon}\right)}\right\rangle\right]
$$

In addition for concentrated force acting at the point $\left(x_{1 p}, x_{2 p}\right)$ without motion, the analytical solution for the deflection in time domain can be obtained by

$$
w(t)=\frac{4 q_{0}}{\pi^{4}} \sum_{m=1}^{\infty} \sum_{n=1}^{\infty} \frac{\sin \frac{m \pi x_{1 p}}{a_{1}} \sin \frac{m \pi x_{2 p}}{a_{2}} \sin \frac{m \pi x_{1}}{a_{1}} \sin \frac{m \pi x_{2}}{a_{2}}}{\left(\frac{m^{2}}{a_{1}^{2}}+\frac{n^{2}}{a_{2}^{2}}\right)^{2}}\left[1+\frac{e^{\alpha t}}{\beta}(\alpha \sin \beta t-\beta \cos \beta t)\right]
$$

if $c<\frac{2 \sqrt{6(1-v)} D \pi^{2}}{c_{2} h}\left(\frac{m^{2}}{a_{1}^{2}}+\frac{n^{2}}{a_{2}^{2}}\right)$ and otherwise

$$
\begin{aligned}
& w(t)=\frac{4 q_{0}}{\pi^{4}} \sum_{m=1}^{\infty} \sum_{n=1}^{\infty} \frac{\sin \frac{m \pi x_{1 p}}{a_{1}} \sin \frac{m \pi x_{2 p}}{a_{2}} \sin \frac{m \pi x_{1}}{a_{1}} \sin \frac{m \pi x_{2}}{a_{2}}}{\left(\frac{m^{2}}{a_{1}^{2}}+\frac{n^{2}}{a_{2}^{2}}\right)^{2}}\left[1+\frac{1}{2 \sqrt{\lambda^{2}-4 \varepsilon}}\left\langle\left(\lambda-\sqrt{\lambda^{2}-4 \varepsilon}\right) \times\right.\right. \\
& \left.\left.e^{-\frac{t}{2}\left(\lambda+\sqrt{\lambda^{2}-4 \varepsilon}\right)}-\left(\lambda+\sqrt{\lambda^{2}-4 \varepsilon}\right) e^{-\frac{t}{2}\left(\lambda-\sqrt{\lambda^{2}-4 \varepsilon}\right)}\right\rangle\right]
\end{aligned}
$$




\section{References}

[1] Tlusty J, MacNeil P. Dynamics of cutting forces in end milling. Annals of the CIRP 1975; 24(1): 21-25.

[2] Altintas Y, Engin S. Generalized modeling of mechanics and dynamics of milling cutters. CIRP Annals - Manufacturing Technology 2001; 50(1): 25-30.

[3] Gradisek J, Kalveram M, Weinert K. Mechanistic identification of specific force coefficients for a general end mill. International Journal of Machine Tools and Manufacture 2004; 44(4): 401-414.

[4] Altintas Y. Modeling approaches and software for predicting the performance of milling operations at MAL-UBC. Machining Science and Technology 2000; 4(3): 445-478.

[5] Ratchev S, Liu S, Huang W, Becker AA. An advanced FEA based force induced error compensation strategy in milling. International Journal of Machine Tools and Manufacture 2006; 46(5): 542-551.

[6] Tsai JS, Liao CL. Finite-element modeling of static surface errors in the peripheral milling of thin-walled workpieces. Journal of Materials Processing Technology 1999; 94(2): 235-246.

[7] Bravo U, Altuzarra O, López de Lacalle LN, Sánchez JA, Campa FJ. Stability limits of milling considering the flexibility of the workpiece and the machine. International Journal of Machine Tools and Manufacture 2005; 45(15): 1669-1680.

[8] Cheng AHD, Cheng DT. Heritage and early history of the boundary element method. Engineering Analysis with Boundary Element Methods 2005; 29: 268-302.

[9] Vander Weeën F. Application of the boundary integral equation method to Reissner's plate model. Int. J. Numer. Methods Engng 1982; 18: 1-10.

[10] Ante H. Static and dynamic analysis of Reissner-Mindlin plates, Boundary Element Analysis of Plates and Shells, Ed. D.E. Beskos, Springer-Verlag, Berlin, Heideberg, 312-340, 1991.

[11] Ante H, Cheng W. Vibration of Reissner Plates by BEM and D/BEM, Boundary Elements:Fundamental \& Applications, Ed. S. Kobayashi and N. Nishimura, IABEM-91 Int. Symposium on Boundary Element Method, Springer-Verlag, Berlin, Heideberg, 30-39, 1992. [12] Wen PH, Aliabadi MH. Boundary element frequency domain formulatio for dynamic analysis of Mindlin plates. Int. J. Numer. Meth. Engng 2006; 67:1617-1640. 
[13] Wen PH. The fundamental solution of Mindlin plates resting on an elastic foundation in the Laplace domain and its applications 2008; 45:1032-1050.

[14] Chen W, Shen ZJ, Shen LJ, Yuan GW. General solutions and fundamental solutions of varied orders to the vibrational thin, the Berger, and the Winkler plates, Engineering Analysis with Boundary Elements 2005; 29:699-702.

[15] Atluri SN. The Meshless Method (MLPG) for Domain and BIE Discretizations. Forsyth: GA, USA, Tech Science Press, 2004.

[16] Kupradze VD, Aleksidze MA. The method of the fundamental equations for the approximation of certain boundary value problems. USSR Computational Mathematics and Mathematical Physics 1964; 4: 82-126.

[17] Wen PH. Point intensity method of solving circular plate resting on elastic subgrade. Engng Mech. 1987; 4 (2): 18-26.

[18] Wen PH. Boundary collocation method for rectangular plate with free corners resting on the elastic foundation. Shanghai Mechanics 1989; 10(1): 71-77.

[19] Marin L, Lesnic D. The method of fundamental solutions for the Cauchy problem in two-dimensional linear elasticity. Int. J. Solids and Struct. 2004; 41: 3425-3438.

[20] Chen CS, Golberg MA, Hon YC. The method of fundamental solutions and QuasiMonte-Carlo method for diffusion equations. Int. J. Numer. Mech. Engng. 1998; 43: 14211435.

[21] Davies MA, Balachandran B. Impact dynamics in milling of thin-walled structures, Nonlinear Dynamics 2000; 22: 375-392.

[22] Sih GC, Mechanics of Fracture, Vol 3. Noordhoff International Publishing: Leyden, 1978.

[23] Aliabadi MH. The Boundary Element Method, Vol 2: Applications in Solids and Structures. Wiley: New York, 2002.

[24] Durbin F. Numerical inversion of Laplace transforms: an efficient improvement to Dubner and Abate's method. Comput. J. 1974; 17(4): 371-376.

[25] Wen PH, Aliabadi MH, Rooke DP. The influence of elastic waves on dynamic stress intensity factors (three dimensional problem). Archive of Applied Mechanics 1996; 66(6): 385-384. 


\section{Legends}

Figure 1. Sign convention of displacement and internal forces.

Figure 2. (a) Convention of traction on the boundary; (b) collocation (P) and source ( $\mathbf{Q})$ points.

Figure 3. A moving force with speed $V$ in the region $x_{1} \in[0, b]$ and observing points $\mathrm{A}$ and B.

Figure 4. Normalized moment $M_{22}(t) / P$ and shear force $Q_{2}(t) a / P$ at observing points A and B due to a moving force $P$ with speed $V=0.2 c_{2}$.

Figure 5. Normalized moment $M_{22}(t) / P$ and shear force $Q_{2}(t) a / P$ at observing points A and B due to a moving force $P$ with speed $V=0.5 c_{2}$.

Figure 6. Normalized moment $M_{22}(t) / P$ and shear force $Q_{2}(t) a / P$ at observing points A and $\mathrm{B}$ due to a moving force $P$ with speed $V=c_{2}$.

Figure 7. Simply supported square $\left(a_{2} / a_{1}=1\right)$ subjected to the uniform pressure load $q_{0}$ and distributions of collocation and source points: (a) geometry; (b) distributions of source and collocation points.

Figure 8. Normalized moment $w_{3} D / q_{0} a^{4}$ at centre of simply supported plate subjected to a uniform load $q_{0} H(t)$.

Figure 9. Rectangular plate with three edges built in and one edge free $\left(a_{1} / a_{2}=1\right)$ subjected to a uniform pressure load $q_{0}$, a concentrated force at the centre of plate and a moving force. Figure 10. Comparisons of the deflections $w_{3}(\mathrm{~mm})$ at points $\mathrm{B}$ and $\mathrm{O}$ for a plate with three edges built in and one edge free under uniform load $q_{0} H(t)$ without damping.

Figure 11. Comparisons of the deflections $w_{3}(\mathrm{~mm})$ at points $\mathrm{B}$ and $\mathrm{O}$ for a plate with three edges built in and one edge free under uniform load $q_{0} H(t)$ with damping.

Figure 12. Normalized moment $w_{3} \mathrm{D} / \mathrm{Pa}^{2}$ at observing points $\mathrm{B}$ and $\mathrm{D}$ for a plate with three edges buildt in and one edge free under a concentrated force acting at the centre of plate $P H(t)$ while the speed of moving force $V / c_{2}=0.1$ : (a) without damping; (b) with damping $c=20 D / c_{2} a^{2} h$. 
Figure 13. Normalized moment $w_{3} \mathrm{D} / \mathrm{Pa}^{2}$ at observing points $\mathrm{B}$ and $\mathrm{D}$ for a plate with three edges buildt in and one edge free under a concentrated force acting at the centre of plate $P H(t)$ while the speed of moving force $V / c_{2}=0.2$ : (a) without damping; (b) with damping $c=20 D / c_{2} a^{2} h$. 

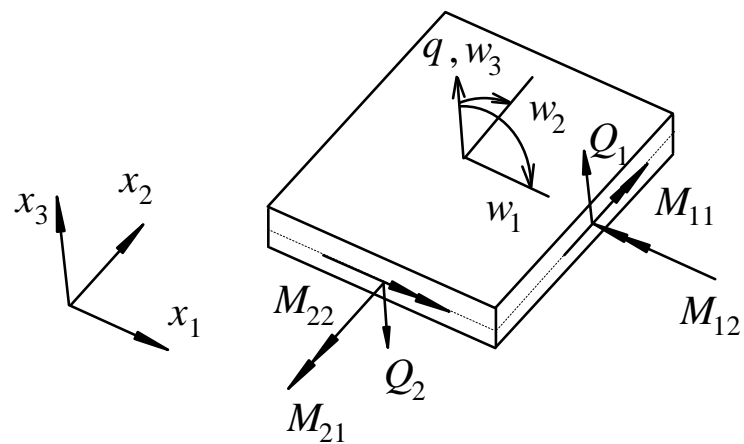

Figure 1. Sign convention of displacement and internal forces.
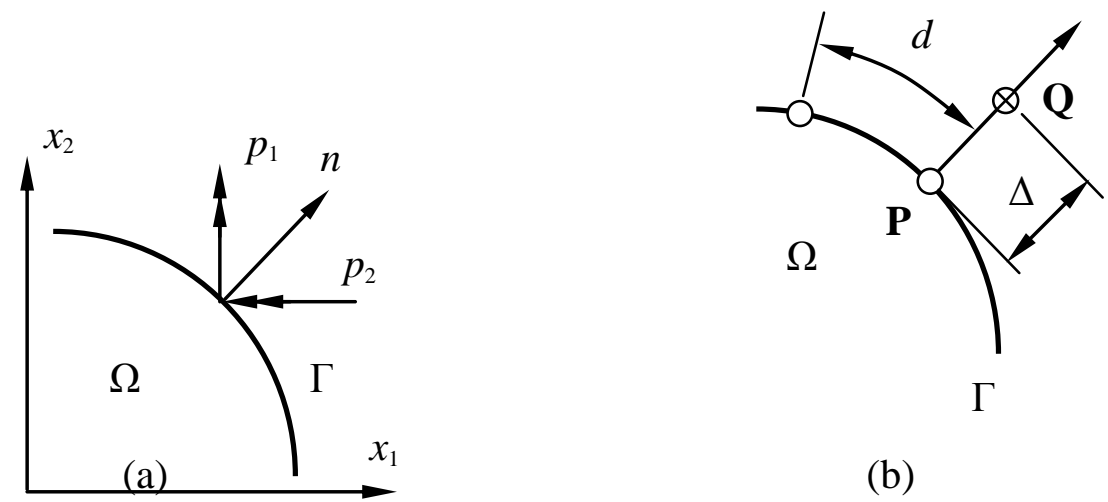

(b)

Figure 2. (a) Convention of traction on the boundary; (b) collocation (P) and source $(\mathbf{Q})$ points. 


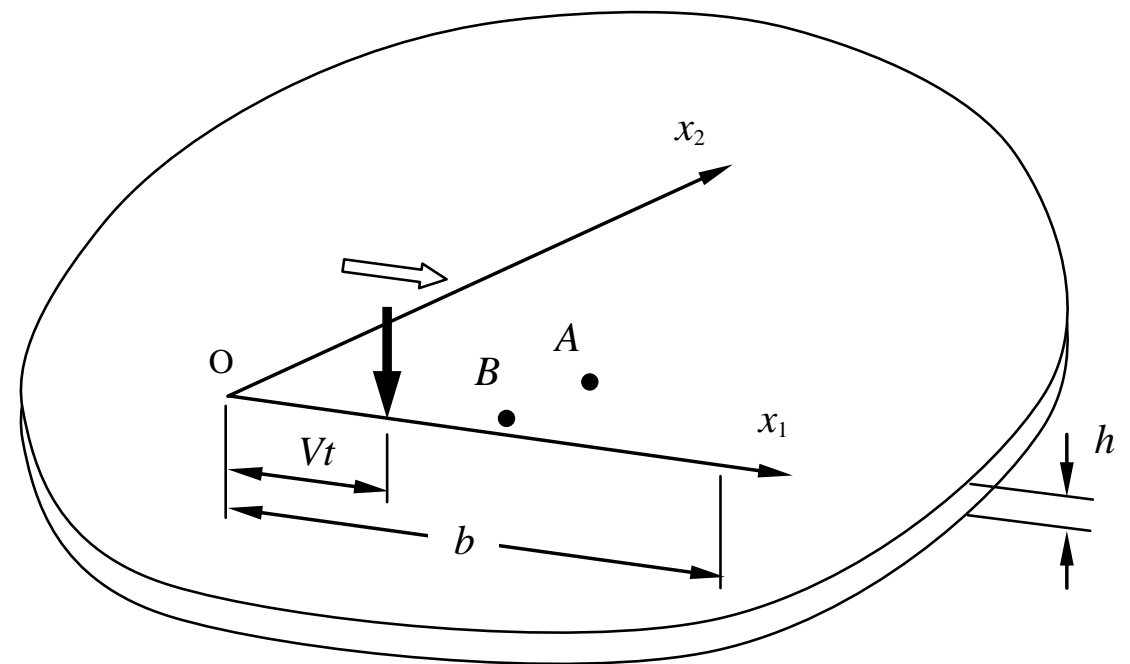

Figure 3. A moving force with speed $V$ in the region $x_{1} \in[0, b]$ and observing points A and $\mathrm{B}$. 

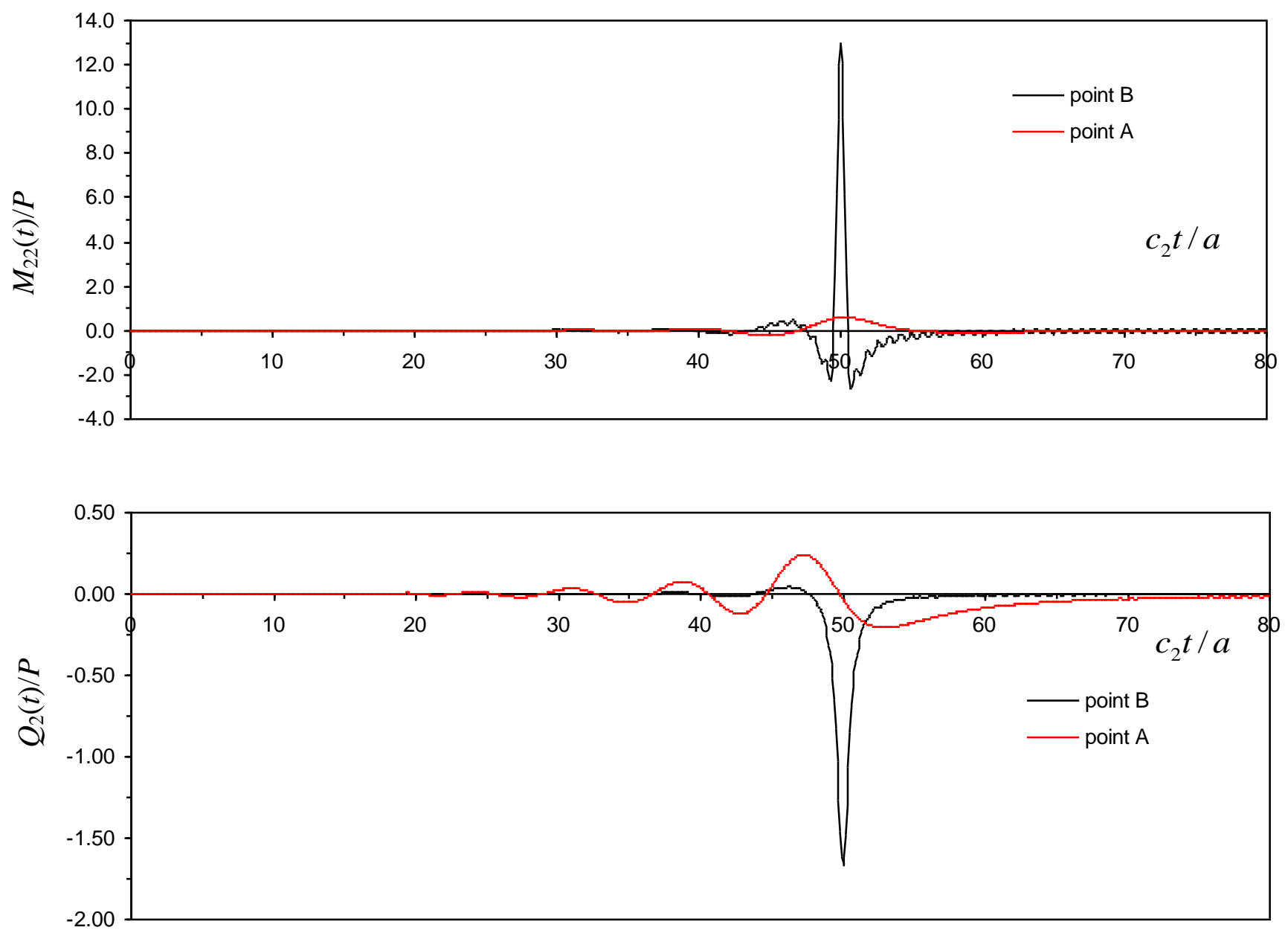

Figure 4. Normalized moment $M_{22}(t) / P$ and shear force $Q_{2}(t) a / P$ at observing points A and B due to a moving force $P$ with speed $V=0.2 c_{2}$. 

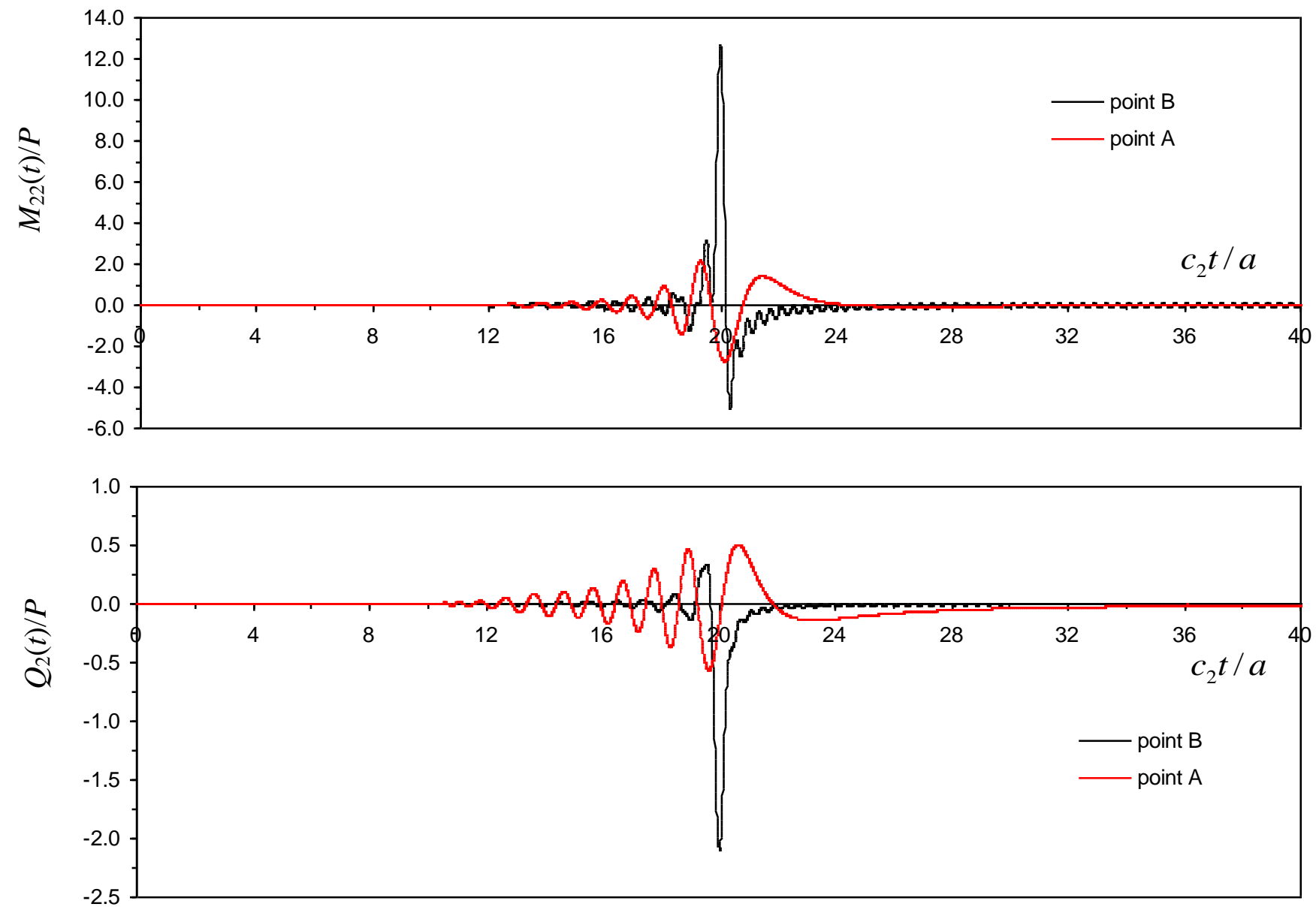

Figure 5. Normalized moment $M_{22}(t) / P$ and shear force $Q_{2}(t) a / P$ at observing points A and B due to a moving force $P$ with speed $V=0.5 c_{2}$. 

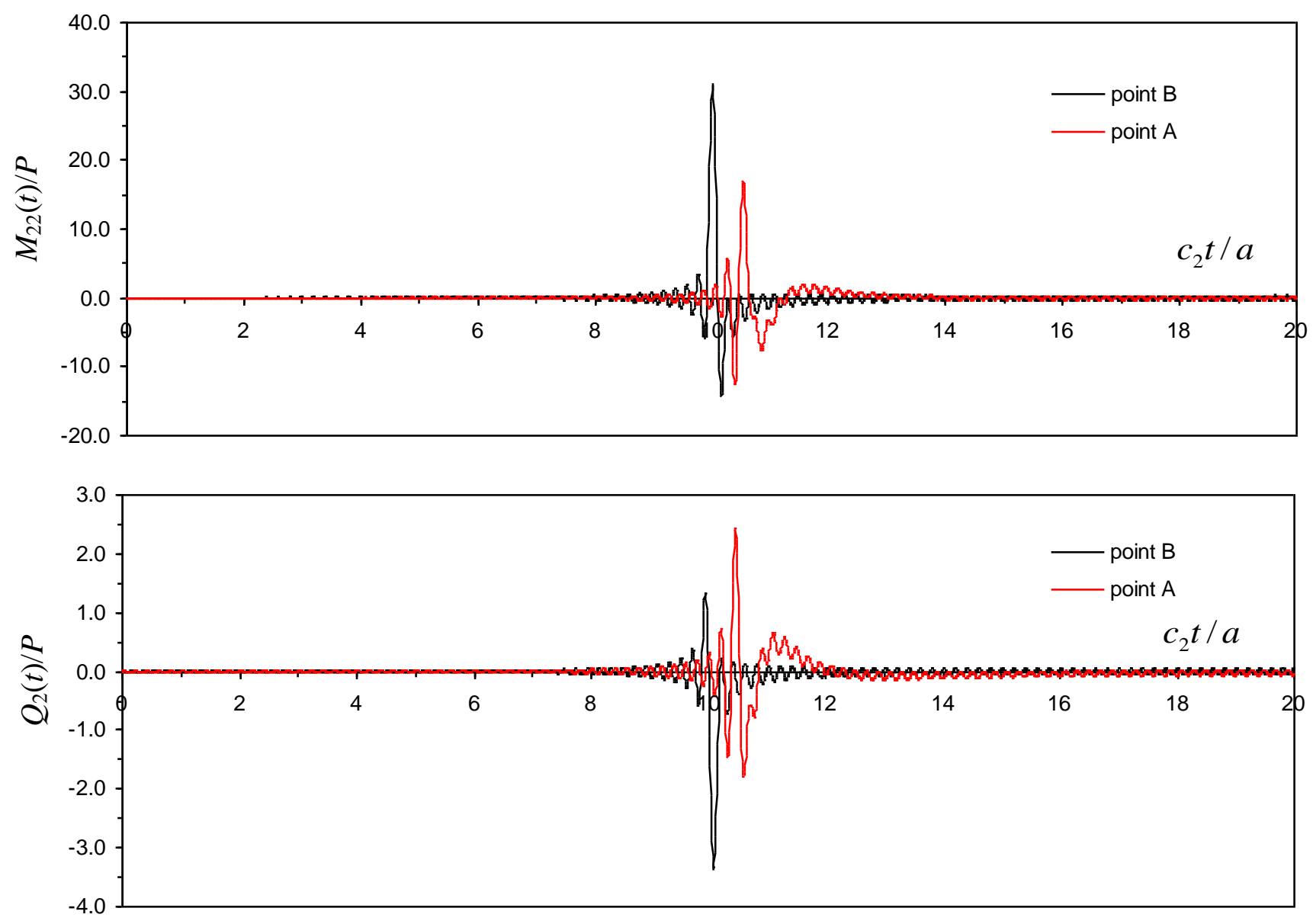

Figure 6. Normalized moment $M_{22}(t) / P$ and shear force $Q_{2}(t) a / P$ at observing points A and B due to a moving force $P$ with speed $V=c_{2}$. 


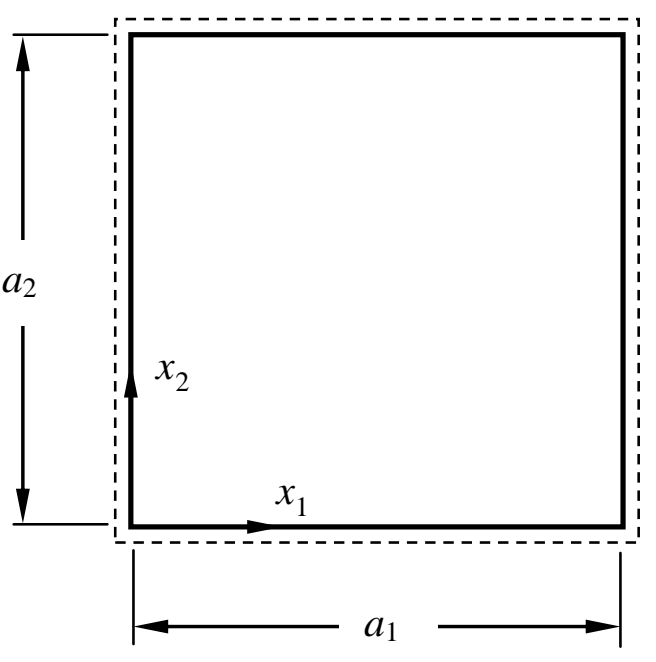

(a)

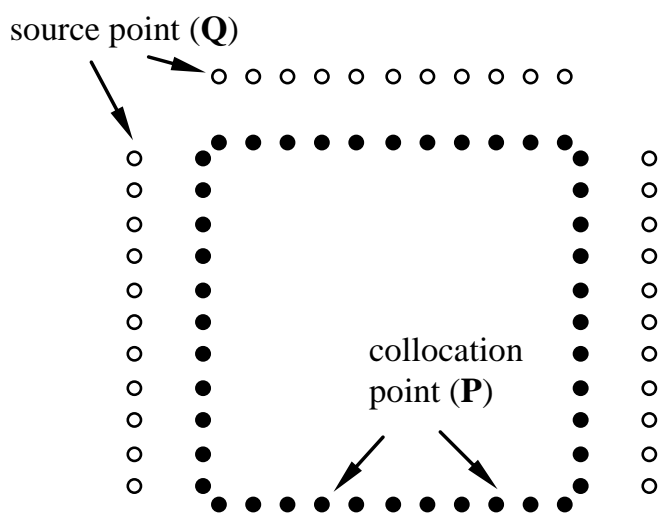

00000000000

(b)

Figure 7. Simply supported square $\left(a_{2} / a_{1}=1\right)$ subjected to the uniform pressure load $q_{0}$ and distributions of collocation and source points: (a) geometry; (b) distributions of source and collocation points. 

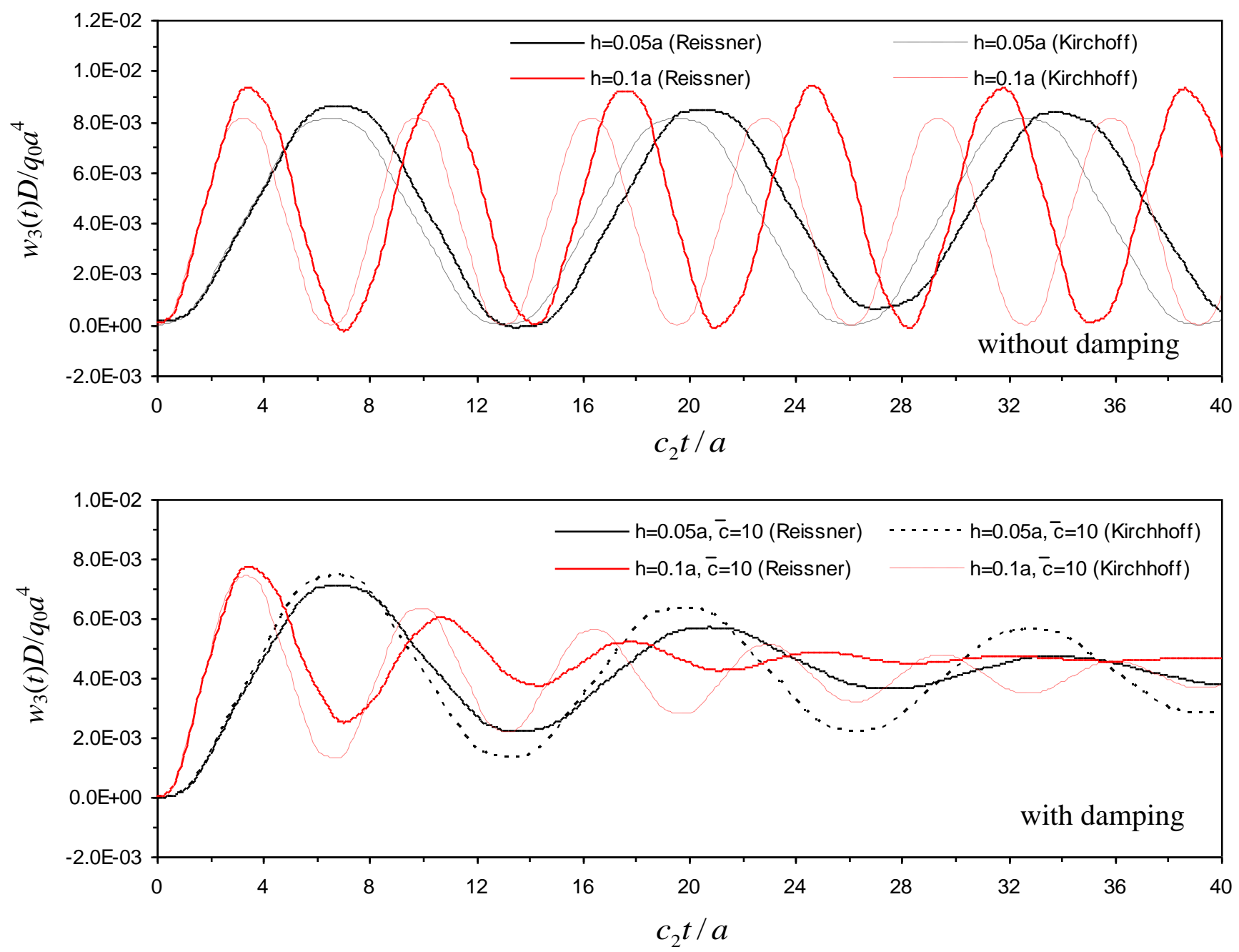

Figure 8. Normalized moment $w_{3} D / q_{0} a^{4}$ at centre of simply supported plate subjected to a uniform load $q_{0} H(t)$. 


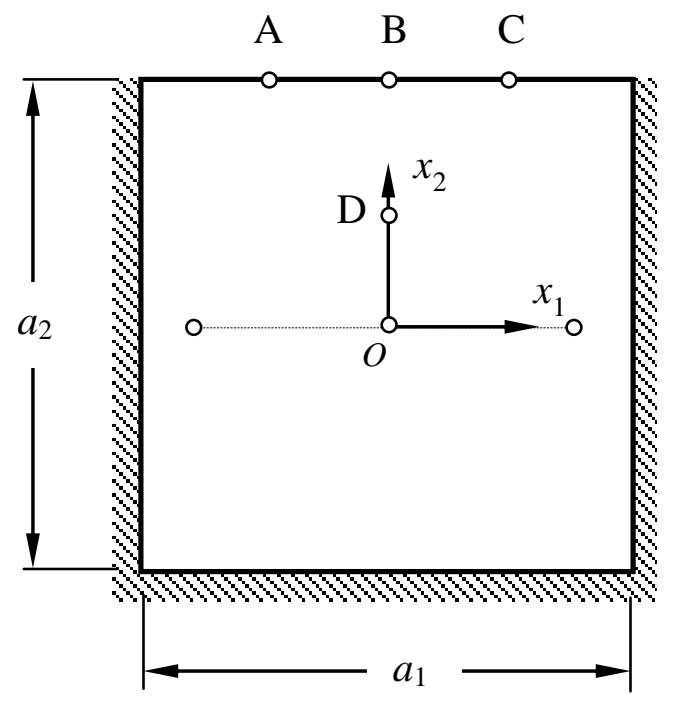

Figure 9. Rectangular plate with three edges built in and one edge free $\left(a_{1} / a_{2}=1\right)$ subjected to a uniform pressure load $q_{0}$, a concentrated force at the centre of plate and a moving force. 


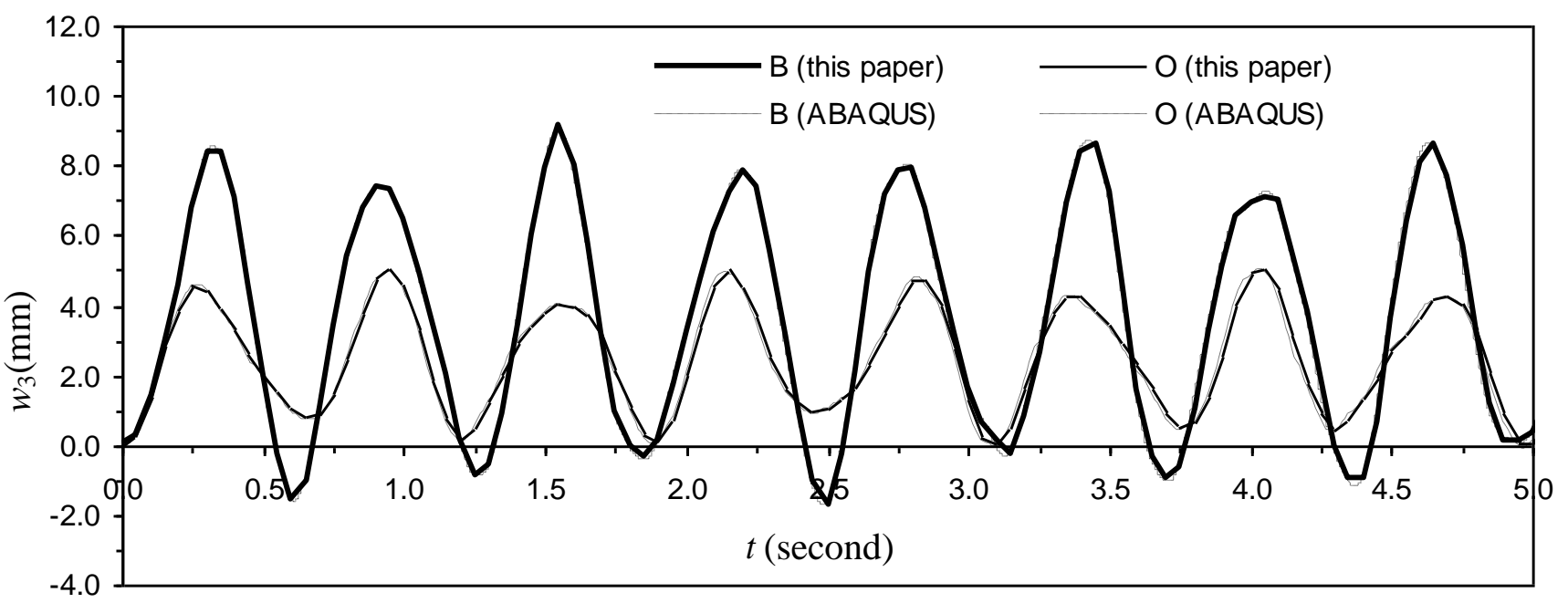

Figure 10. Comparisons of the deflections $w_{3}(\mathrm{~mm})$ at points $\mathrm{B}$ and $\mathrm{O}$ for a plate with three edges built in and one edge free under uniform load $q_{0} H(t)$ without damping.
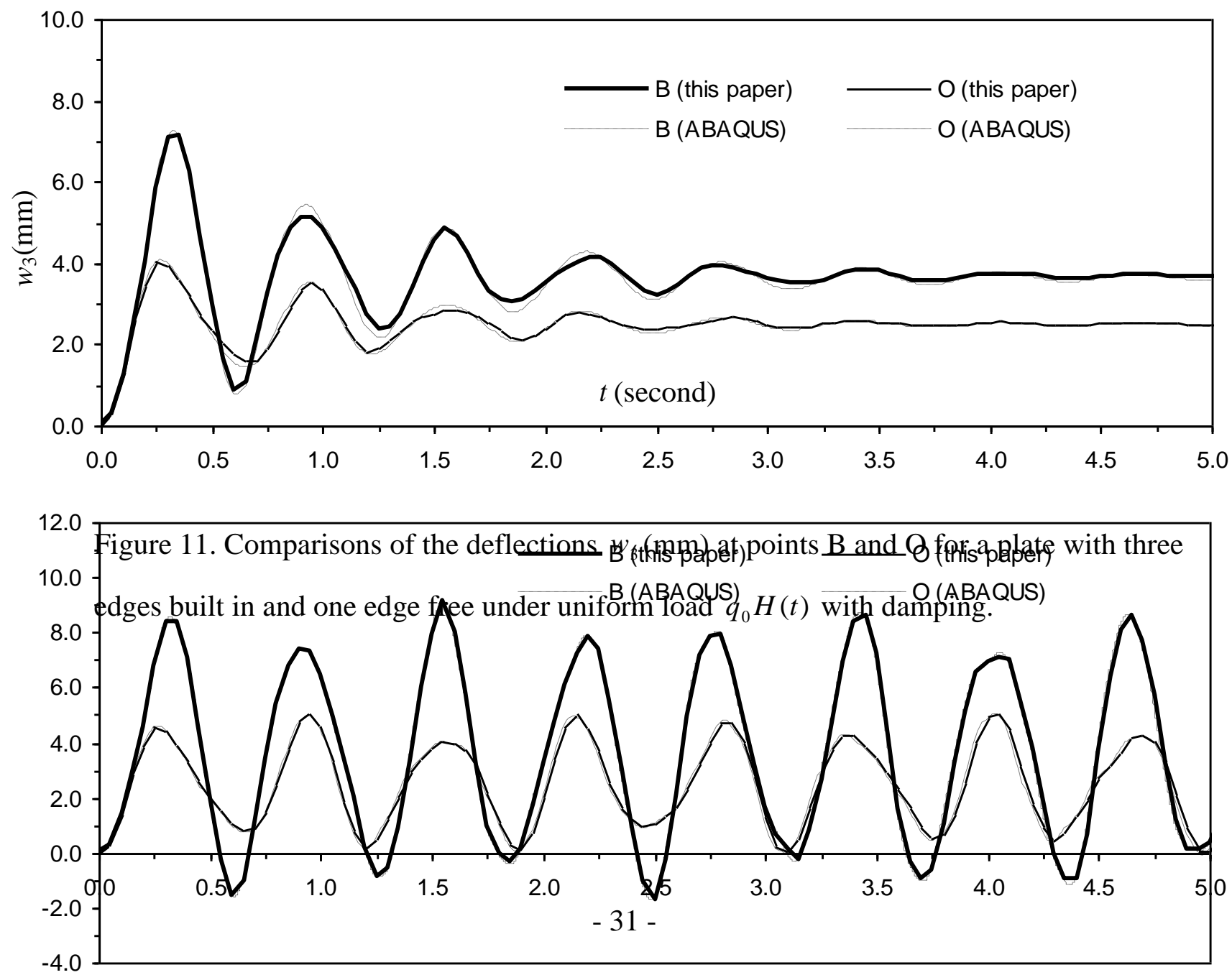

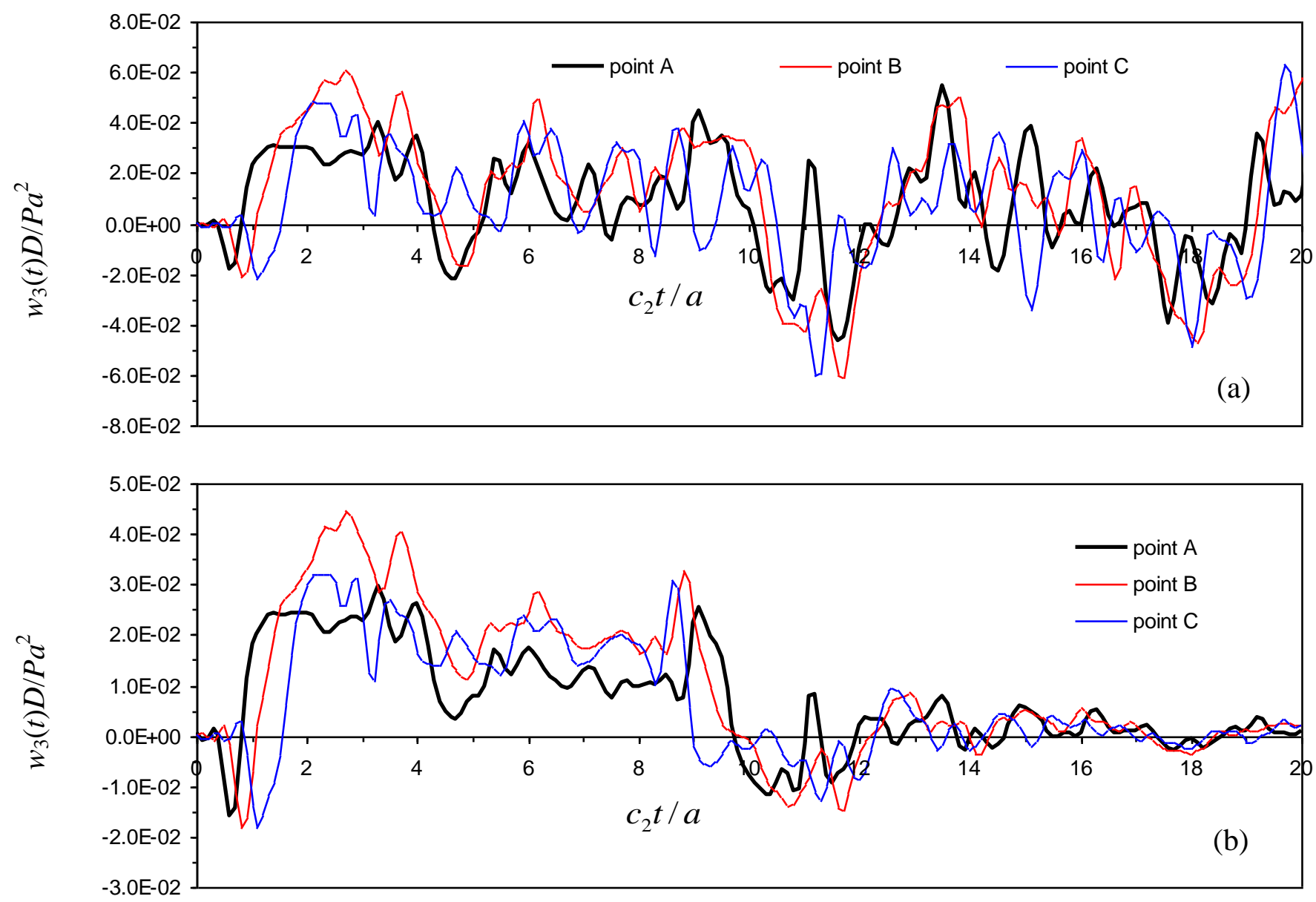

Figure 12. Normalized moment $w_{3} \mathrm{D} / \mathrm{Pa}^{2}$ at observing points $\mathrm{B}$ and $\mathrm{D}$ for a plate with three edges built in and one edge free under a concentrated force $P H(t)$ acting at the centre of platewhile the speed of moving force $V / c_{2}=0.1$ : (a) without damping; (b) with damping $c=20 \mathrm{D} / \mathrm{c}_{2} a^{2} h$. 

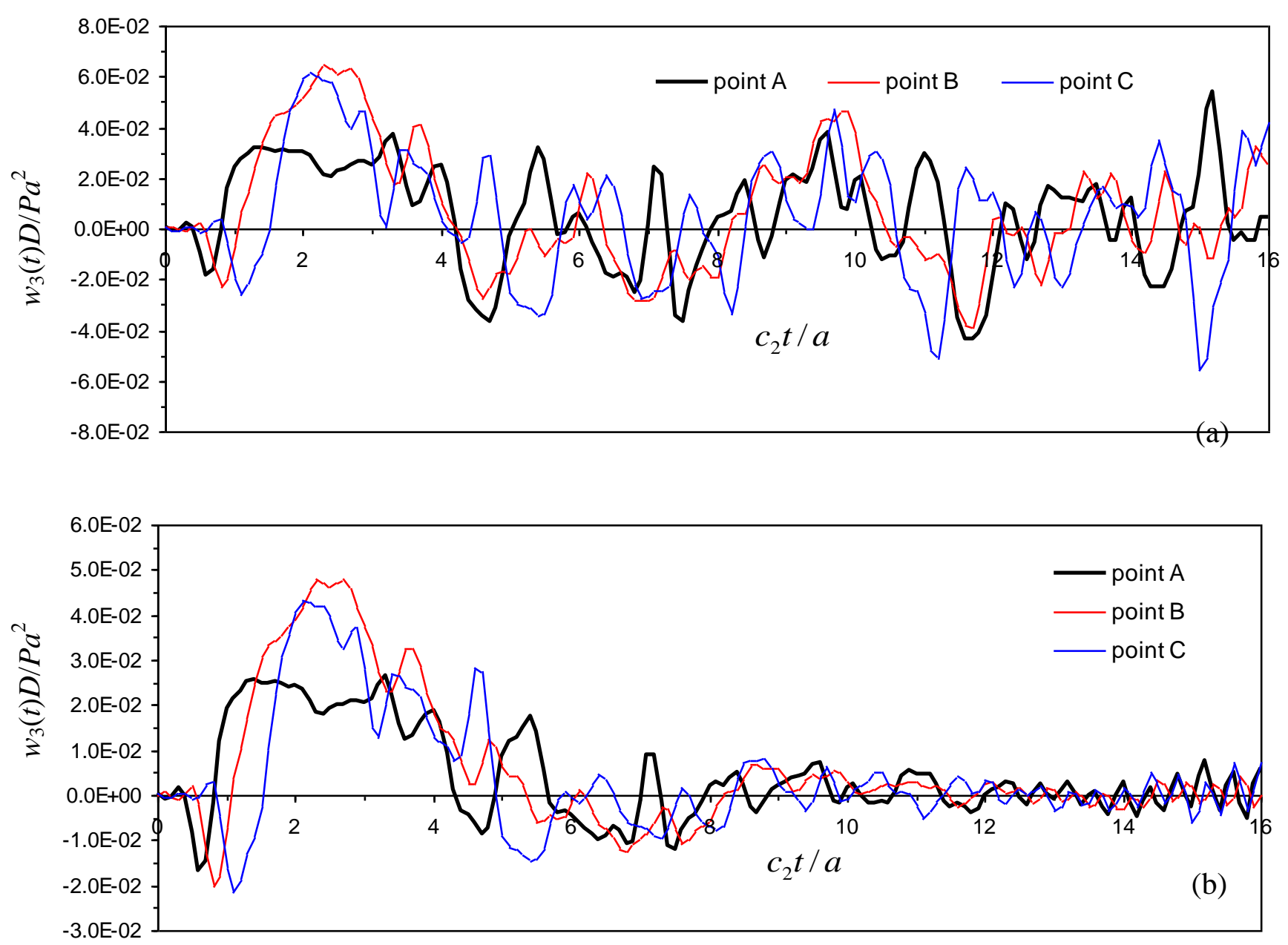

Figure 13. Normalized moment $w_{3} \mathrm{D} / \mathrm{Pa}^{2}$ at observing points $\mathrm{B}$ and $\mathrm{D}$ for a plate with three edges built in and one edge free under a concentrated force $P H(t)$ acting at the centre of plate while the speed of moving force $V / c_{2}=0.2$ : (a) without damping; (b) with damping $c=20 D / c_{2} a^{2} h$. 